\title{
The Odd Solutions of Equations Involving Euler-Like Function
}

\author{
Jiaxin Wu, Zhongyan Shen* \\ Department of Mathematics, Zhejiang International Studies University, Hangzhou, China \\ Email: *huanchenszyan@163.com
}

How to cite this paper: Wu, J.X. and Shen, Z.Y. (2021) The Odd Solutions of Equations Involving Euler-Like Function. $A d$ vances in Pure Mathematics, 11, 440-446. https://doi.org/10.4236/apm.2021.115030

Received: April 16, 2021

Accepted: May 22, 2021

Published: May 25, 2021

Copyright $\odot 2021$ by author(s) and Scientific Research Publishing Inc. This work is licensed under the Creative Commons Attribution International License (CC BY 4.0).

http://creativecommons.org/licenses/by/4.0/

\begin{abstract}
$\varphi_{e}(n)$ is a function similar to Euler function $\varphi(n)$. We discussed and obtained all the odd solutions of the equations $\varphi_{e}(x y)=\varphi_{e}(x)+2 \varphi_{e}(y)$, $\varphi_{e}(x y)=2 \varphi_{e}(x)+3 \varphi_{e}(y)$ and $\varphi_{e}(x y z)=\varphi_{e}(x)+\varphi_{e}(y)+\varphi_{e}(z)$ by using the methods and techniques of elementary number theory.
\end{abstract}

\section{Keywords}

Euler-Like Function, Diophantine Equation, Odd Solutions

\section{Introduction}

Euler function is an important function in number theory. For the past few years, many scholars have discussed the solvability of equations involving Euler function and achieved some results. The solvability of the equation $\varphi(x y)=k_{1} \varphi(x)+k_{2} \varphi(y)$ for given values of $k_{1}, k_{2}$ has been discussed in the literature [1] [2] [3] [4] [5]. The positive integer solutions of the equation $\varphi(x y z)=k_{1} \varphi(x)+k_{2} \varphi(y)+k_{3} \varphi(z)$ for given values of $k_{1}, k_{2}, k_{3}$ have been discussed in the literature [6] [7] [8] [9].

$\varphi_{e}(n)$, which is extremely similar to Euler function, is defined in the literature [10]. Function $\varphi_{e}(n)$ is the number of positive integers $i$ not greater than $n$, such that $\operatorname{gcd}(i(i+1), n)=1$, i.e.,

$$
\varphi_{e}(n)=\sum_{\substack{i=1 \\(i(i+1), n)=1}}^{n} 1 .
$$

Define $\varphi_{e}(1)=1$ for the convenience. When the positive integer $n>1$, let $n=\prod_{i=1}^{k} p_{i}^{a_{i}}$. Then [10]

${ }^{*}$ Corresponding author. 


$$
\varphi_{e}(n)=\prod_{i=1}^{k} p_{i}^{a_{i}-1}\left(p_{i}-2\right) .
$$

By the definition of $\varphi_{e}(n)$, we have $\varphi_{e}(n)=0$ if $n$ is even. So we are only interested in the case when $n$ is odd. Here we consider the odd solutions of the equation containing $\varphi_{e}(n)$ and we obtain the following results.

Theorem 1 For the equation

$$
\varphi_{e}(x y)=\varphi_{e}(x)+2 \varphi_{e}(y),
$$

there are three odd solutions, which are $(x, y)=(5,9),(9,5),(3,3)$.

For the equation

$$
\varphi_{e}(x y)=2 \varphi_{e}(x)+3 \varphi_{e}(y),
$$

there are ten odd solutions, which are $(x, y)=(11,5),(11,9),(11,15),(27,5),(33,5),(9,3),(15,3),(5,5),(5,15),(15,5)$.

Theorem 2 For the equation

$$
\varphi_{e}(x y z)=\varphi_{e}(x)+\varphi_{e}(y)+\varphi_{e}(z),
$$

there are three odd solutions, which are $(x, y, z)=(1,3,3),(3,1,3),(3,3,1)$.

\section{Lemmas}

Lemma 1 [10] If the positive integers $x$ and $y$ satisfy that $\operatorname{gcd}(x, y)=1$, then we have

$$
\varphi_{e}(x y)=\varphi_{e}(x) \varphi_{e}(y)
$$

Lemma 2 Let $x \geq 3$ be an odd integer, then $\varphi_{e}(x)$ is odd. Let $x \geq 2$ be an integer, we have $\varphi_{e}(x)<x$.

Proof By the definition of $\varphi_{e}(n)$.

Lemma 3 If $x \mid y$, for any odd positive integer $x$ and positive integer $y$. Then we have $\varphi_{e}(x) \mid \varphi_{e}(y)$.

Proof When $x=1$ and $x\left|y, \varphi_{e}(x)\right| \varphi_{e}(y)$ holds because of $\varphi_{e}(x)=1$.

When $x>1$ and $x \mid y$, suppose that $x=\prod_{i=1}^{k} p_{i}^{a_{i}}, a_{i} \geq 1, i=1,2, \cdots, k$ and

$$
y=\prod_{i=1}^{k} p_{i}^{a_{i}+b_{i}} \prod_{i=k+1}^{t} p_{i}^{c_{i}}, \quad b_{i} \geq 0, \quad c_{i} \geq 1
$$

(Especially when $t<k+1$, suppose that $y=\prod_{i=1}^{k} p_{i}^{a_{i}+b_{i}}$, we can easily prove the lemma by calculation.) Then

$$
\begin{gathered}
\varphi_{e}(x)=\prod_{i=1}^{k} p_{i}^{a_{i}-1}\left(p_{i}-2\right), \\
\varphi_{e}(y)=\prod_{i=1}^{k} p_{i}^{a_{i}+b_{i}-1}\left(p_{i}-2\right) \prod_{i=k+1}^{t} p_{i}^{c_{i}-1}\left(p_{i}-2\right),
\end{gathered}
$$

Obviously $\varphi_{e}(x) \mid \varphi_{e}(y)$.

Lemma 4 Let $d=\operatorname{gcd}(x, y)$ be an odd integer, then

$$
\varphi_{e}(x y)=\frac{d \varphi_{e}(x) \varphi_{e}(y)}{\varphi_{e}(d)} .
$$


Proof When $d=1$, by lemma 1 we have

$$
\varphi_{e}(x y)=\varphi_{e}(x) \varphi_{e}(y)=\frac{d \varphi_{e}(x) \varphi_{e}(y)}{\varphi_{e}(d)} .
$$

When $d>1$, let $d=\prod_{i=1}^{k} p_{i}^{a_{i}}, x=\prod_{i=1}^{k} p_{i}^{a_{i}+b_{i}} \prod_{i=k+1}^{t} p_{i}^{d_{i}}$,

$$
y=\prod_{i=1}^{k} p_{i}^{a_{i}+c_{i}} \prod_{i=k+1}^{s} q_{i}^{e_{i}}, a_{i} \geq 1, b_{i}, c_{i} \geq 0, d_{i}, e_{i} \geq 1 .
$$

(Especially when $t<k+1, \quad x=\prod_{i=1}^{k} p_{i}^{a_{i}+b_{i}}$. When $s<k+1, \quad y=\prod_{i=1}^{k} p_{i}^{a_{i}+c_{i}}$. We can easily prove the lemma by calculation.) Then according to

$$
\varphi_{e}(x y)=\prod_{i=i}^{k} p_{i}^{2 a_{i}+b_{i}+c_{i}-1}\left(p_{i}-2\right) \prod_{i=k+1}^{t} p_{i}^{d_{i}-1}\left(p_{i}-2\right) \prod_{i=k+1}^{s} q_{i}^{e_{i}-1}\left(q_{i}-2\right),
$$

$$
\begin{aligned}
\frac{d \varphi_{e}(x) \varphi_{e}(y)}{\varphi_{e}(d)} & =\frac{\prod_{i=1}^{k} p_{i}^{a_{i}} \prod_{i=1}^{k} p_{i}^{a_{i}+b_{i}-1}\left(p_{i}-2\right) \prod_{i=k+1}^{t} p_{i}^{d_{i}-1}\left(p_{i}-2\right) \prod_{i=1}^{k} p_{i}^{a_{i}+c_{i}-1}\left(p_{i}-2\right) \prod_{i=k+1}^{s} q_{i}^{e_{i}-1}\left(q_{i}-2\right)}{\prod_{i=1}^{k} p_{i}^{a_{i}-1}\left(p_{i}-2\right)} \\
& =\prod_{i=1}^{k} p_{i}^{a_{i}} \prod_{i=i}^{k} p_{i}^{a_{i}+b_{i}+c_{i}-1}\left(p_{i}-2\right) \prod_{i=k+1}^{t} p_{i}^{d_{i}-1}\left(p_{i}-2\right) \prod_{i=k+1}^{s} q_{i}^{e_{i}-1}\left(q_{i}-2\right) \\
& =\prod_{i=i}^{k} p_{i}^{2 a_{i}+b_{i}+c_{i}-1}\left(p_{i}-2\right) \prod_{i=k+1}^{t} p_{i}^{d_{i}-1}\left(p_{i}-2\right) \prod_{i=k+1}^{s} q_{i}^{e_{i}-1}\left(q_{i}-2\right),
\end{aligned}
$$

we have

$$
\varphi_{e}(x y)=\frac{d \varphi_{e}(x) \varphi_{e}(y)}{\varphi_{e}(d)} .
$$

This completes the proof of Lemma 4.

Lemma 5 The odd solutions of the equation $\varphi_{e}(x)=1$ are $x=1,3$.

The odd solutions of the equation $\varphi_{e}(x)=3$ are $x=5,9,15$.

The odd solutions of the equation $\varphi_{e}(x)=5$ are $x=7,21$.

The odd solutions of the equation $\varphi_{e}(x)=9$ are $x=11,27,33,45$.

Proof Here we take the equation $\varphi_{e}(x)=1$ for example. The other three equations can be obtained similarly.

If $x=1, \varphi_{e}(x)=1$. It is easy to see that $x=1$ is the solution of the equation.

If $x>1$, suppose that $x=\prod_{i=1}^{k} p_{i}^{a_{i}}, a_{i} \geq 1, i=1,2, \cdots, k$, then

$$
\varphi_{e}(x)=\prod_{i=1}^{k} p_{i}^{a_{i}-1}\left(p_{i}-2\right)=1 .
$$

Thus we have $\left(p_{i}-2\right) \mid 1$, and then $p_{i}=3$. Let $x=3^{\alpha}$. Then

$$
\varphi_{e}(x)=3^{\alpha-1} \cdot 1=1 \text {. }
$$

Hence $\alpha=1$ and then $x=3$.

So we obtain the solutions of $\varphi_{e}(x)=1$ are $x=1,3$.

This completes the proof of Lemma 5 .

\section{Proof of Theorems}

\subsection{Proof of Theorem 1}

Proof Let $d=\operatorname{gcd}(x, y)$. When $d$ is even, $\varphi_{e}(d)=0,(1)$ holds. So there are 
infinity even solutions of (1). Therefore we only consider the odd solutions of it. Since $d|x, d| y$, by Lemma 3, we have

$$
\varphi_{e}(d)\left|\varphi_{e}(x), \varphi_{e}(d)\right| \varphi_{e}(y) \text {. }
$$

Thus there exist $a, b \in Z^{+}$, such that

$$
\varphi_{e}(x)=a \varphi_{e}(d), \varphi_{e}(y)=b \varphi_{e}(d) .
$$

Then,

$$
\varphi_{e}(x)+2 \varphi_{e}(y)=(a+2 b) \varphi_{e}(d)
$$

By Lemma 4, we know that

$$
\varphi_{e}(x y)=\frac{d \varphi_{e}(x) \varphi_{e}(y)}{\varphi_{e}(d)}=\operatorname{abd} \varphi_{e}(d) .
$$

Combining the two equations above,

$$
a b d \varphi_{e}(d)=(a+2 b) \varphi_{e}(d) .
$$

Dividing $a b \varphi_{e}(d)$ both sides, we have

$$
d=\frac{2}{a}+\frac{1}{b}
$$

It is easy to see that the equation has no integer solutions when $d>3$. So we only need to consider the two cases that $d=1,3$.

Case 1 If $d=1$, then $b>1$. (4) can be simplified as

$$
a=2+\frac{2}{b-1}
$$

Therefore its solutions are $\left\{\begin{array}{l}a=4 \\ b=2\end{array},\left\{\begin{array}{l}a=3 \\ b=3\end{array}\right.\right.$.

For $\left\{\begin{array}{l}a=4 \\ b=2\end{array}\right.$, we have $\left\{\begin{array}{l}\varphi_{e}(x)=4 \\ \varphi_{e}(y)=2\end{array}\right.$. By the definition of $\varphi_{e}(x), \varphi_{e}(x)=0$

when $x$ is even. By Lemma 2, when $x$ is odd, $\varphi_{e}(x)$ must be odd. Thus there is no integer solutions.

$$
\text { As for }\left\{\begin{array} { l } 
{ a = 3 } \\
{ b = 3 }
\end{array} \text { , we have } \left\{\begin{array} { l } 
{ \varphi _ { e } ( x ) = 3 } \\
{ \varphi _ { e } ( y ) = 3 }
\end{array} \text { . By Lemma 5, we know that } \left\{\begin{array}{l}
x=5,9,15 \\
y=5,9,15
\end{array}\right.\right.\right. \text {. }
$$

$\operatorname{Bygcd}(x, y)=1$, the odd integer solutions are $(x, y)=(5,9),(9,5)$.

Case 2 If $d=3$, then $\varphi_{e}(d)=1$. The positive integer solution of (4) is $\left\{\begin{array}{l}a=1 \\ b=1\end{array}\right.$.

Then $\left\{\begin{array}{l}\varphi_{e}(x)=1 \\ \varphi_{e}(y)=1\end{array}\right.$. By Lemma 5, we have $\left\{\begin{array}{l}x=1,3 \\ y=1,3\end{array}\right.$. By $\operatorname{gcd}(x, y)=3$, the odd integer solution is $(x, y)=(3,3)$.

In conclusion, we obtain the three odd integer solutions of $\varphi_{e}(x y)=\varphi_{e}(x)+2 \varphi_{e}(y)$, which are $(x, y)=(5,9),(9,5),(3,3)$.

It is similar to get the ten odd integer solutions of (2), which are 
$(x, y)=(11,5),(11,9),(11,15),(27,5),(33,5),(9,3),(15,3),(5,5),(5,15),(15,5)$.

This completes the proof of Theorem 1 .

\subsection{Proof of Theorem 2}

Proof We only consider the odd integer solutions of (3). By Lemma 4,

$$
\varphi_{e}(x y z)=\frac{(x y, z) \varphi_{e}(x y) \varphi_{e}(z)}{\varphi_{e}((x y, z))}=\frac{(x y, z)(x, y) \varphi_{e}(x) \varphi_{e}(y) \varphi_{e}(z)}{\varphi_{e}((x y, z)) \varphi_{e}((x, y))} .
$$

From Lemma 2,

$$
\frac{(x y, z)(x, y) \varphi_{e}(x) \varphi_{e}(y) \varphi_{e}(z)}{\varphi_{e}((x y, z)) \varphi_{e}((x, y))} \geq \varphi_{e}(x) \varphi_{e}(y) \varphi_{e}(z) .
$$

So

$$
\varphi_{e}(x y z) \geq \varphi_{e}(x) \varphi_{e}(y) \varphi_{e}(z) .
$$

Then by (3) we have

$$
\begin{gathered}
\varphi_{e}(x)+\varphi_{e}(y)+\varphi_{e}(z) \geq \varphi_{e}(x) \varphi_{e}(y) \varphi_{e}(z), \\
\varphi_{e}(x)+\varphi_{e}(y) \geq\left(\varphi_{e}(x) \varphi_{e}(y)-1\right) \varphi_{e}(z) \geq \varphi_{e}(x) \varphi_{e}(y)-1,
\end{gathered}
$$

i.e.,

$$
\left(\varphi_{e}(x)-1\right)\left(\varphi_{e}(y)-1\right) \leq 2 .
$$

Since $\varphi_{e}(x)$ and $\varphi_{e}(y)$ are odd, $\left(\varphi_{e}(x)-1\right)\left(\varphi_{e}(y)-1\right)$ must be divisible by 4 . So $\left(\varphi_{e}(x)-1\right)\left(\varphi_{e}(y)-1\right)=0$. Therefore $\varphi_{e}(x)=1$ or $\varphi_{e}(y)=1$. Take $\varphi_{e}(y)=1$ for example, the case $\varphi_{e}(x)=1$ is similarly.

If $\varphi_{e}(y)=1$, then

$$
\varphi_{e}(x y z)=\varphi_{e}(x)+\varphi_{e}(z)+1 \geq \varphi_{e}(x) \varphi_{e}(z),
$$

i.e.,

$$
\left(\varphi_{e}(x)-1\right)\left(\varphi_{e}(z)-1\right) \leq 2 .
$$

Similarly, we can conclude that $\varphi_{e}(z)=1$ or $\varphi_{e}(x)=1$. Take $\varphi_{e}(z)=1$ for example, the case $\varphi_{e}(x)=1$ is similarly. If $\varphi_{e}(z)=1,(3)$ is

$$
\varphi_{e}(x y z)=2+\varphi_{e}(x) \text {. }
$$

By $\left\{\begin{array}{l}\varphi_{e}(y)=1 \\ \varphi_{e}(z)=1\end{array}\right.$ and Lemma 5, we have $\left\{\begin{array}{l}x=1,3 \\ y=1,3\end{array}\right.$.

If $\left\{\begin{array}{l}y=1 \\ z=1\end{array}\right.$, we have $\varphi_{e}(x)=2+\varphi_{e}(x)$ by (5). Obviously there is no solution.

If $\left\{\begin{array}{l}y=1 \\ z=3\end{array}\right.$, we have $\varphi_{e}(3 x)=2+\varphi_{e}(x)$ by (5). If $(3, x)=1$, then by Lemma 1, we have $\varphi_{e}(3 x)=\varphi_{e}(x)=2+\varphi_{e}(x)$, there is no solution. If $3 \mid x$, by Lemma 4 and Lemma 5 , then we obtain

$$
\varphi_{e}(3 x)=\frac{3 \varphi_{e}(3) \varphi_{e}(x)}{\varphi_{e}(3)}=2+\varphi_{e}(x) .
$$


Then $\varphi_{e}(x)=1$, we have $x=3$ for $3 \mid x$. Therefore, if $y=1, z=3$, the odd solution of $(3)$ is $(x, y, z)=(3,1,3)$.

If $\left\{\begin{array}{l}y=3 \\ z=1\end{array}\right.$, we have $\varphi_{e}(3 x)=2+\varphi_{e}(x)$ by (5). If $(3, x)=1$, then by Lemma 1 , we have $\varphi_{e}(3 x)=\varphi_{e}(x)=2+\varphi_{e}(x)$. There is no solution. If $3 \mid x$, then by Lemma 4 and Lemma 5 , we obtain

$$
\varphi_{e}(3 x)=\frac{3 \varphi_{e}(3) \varphi_{e}(x)}{\varphi_{e}(3)}=2+\varphi_{e}(x) .
$$

Then $\varphi_{e}(x)=1$, we have $x=3$ for $3 \mid x$. Therefore, if $y=3, z=1$, the odd integer solution of $(3)$ is $(x, y, z)=(3,3,1)$.

If $\left\{\begin{array}{l}y=3 \\ z=3\end{array}\right.$, we have $\varphi_{e}(9 x)=2+\varphi_{e}(x)$ by (5). If $(3, x)=1$, then by Lemma 1 and Lemma 5, we have $\varphi_{e}(9 x)=3 \varphi_{e}(x)=2+\varphi_{e}(x)$. Thus $\varphi_{e}(x)=1$, we have $x=1$ for $(3, x)=1$. Thus, if $y=3, z=3$, the odd integer solution of (3) is $(x, y, z)=(1,3,3)$. If $3 \mid x$, by Lemma 4 it is easy to see that

$$
\varphi_{e}(9 x)=\frac{3 \varphi_{e}(3) \varphi_{e}(3 x)}{\varphi_{e}(3)}=3 \varphi_{e}(3 x)=\frac{9 \varphi_{e}(3) \varphi_{e}(x)}{\varphi_{e}(3)}=9 \varphi_{e}(x)=2+\varphi_{e}(x) .
$$

There is no solution.

Above all, we obtain three odd solutions of $\varphi_{e}(x y z)=\varphi_{e}(x)+\varphi_{e}(y)+\varphi_{e}(z)$, which are $(x, y, z)=(1,3,3),(3,1,3),(3,3,1)$.

This completes the proof of Theorem 2 .

\section{Conclusion}

Our method can be used to solve the equations such as $\varphi_{e}(x y)=a \varphi_{e}(x)+b \varphi_{e}(y)$, with $a, b$ the different parity, and $\varphi_{e}(x y z)=a \varphi_{e}(x)+b \varphi_{e}(y)+c \varphi_{e}(z)$, with $a+b+c$ is odd. For a specific equation, it is easy to solve it. Whether it can be used to solve all this kind of equations is what we are interested in.

\section{Founding}

This work is supported by the Natural Science Foundation of Zhejiang Province, Project (No. LY18A010016) and the National Natural Science Foundation of China, Project (No. 12071421).

\section{Conflicts of Interest}

The authors declare no conflicts of interest regarding the publication of this paper.

\section{References}

[1] Sun, C. and Cheng, Z. (2010) Some Kind of Equation Involving Euler Function. Journal of Mathematical Study, 43, 364-369. 
https://doi.org/10.3969/j.issn.1006-6837.2010.04.008

[2] Xu, X. and Xu, X.F. (2016) On Positive Integer Solutions of Euler Equation $\varphi(a b)=2^{k}(\varphi(a)+\varphi(b))$. Journal of Southwest China Normal University (Natural Science Edition), 41, 6-9. https://doi.org/10.13718/j.cnki.xsxb.2016.04.002

[3] Sun, S. (2015) Positive Integer Solutions of an Equation on Euler Function $\varphi(n)$. Journal of Beihua University (Natural Science), 16, 161-164.

[4] Zhang, S. and Xi, X. (2016) Positive Integer Solutions on Equation $\varphi(a b)=k(\varphi(a)+\varphi(b))$. Journal of Nanjing University (Natural Science Edition), 39, 41-47. https://doi.org/10.3969/j.issn.1001-4616.2016.01.007

[5] Zhang, M. and Gao, L. (2018) The Positive Integer Solutions of Euler Function $\varphi(m n)=2^{2} \times 3(\varphi(m)+\varphi(n))$. Journal of Yanan University (Natural Science Edition), 37, 5-9. https://doi.org/10.13876/j.cnki.ydnse.2018.02.005

[6] Zhang, S. and Du, X. (2015) The Positive Integer Solutions of an Equation involving the Euler Function. Journal of Central China Normal University (Nat. Sci.), 49, 497501. https://doi.org/10.3969/j.issn.1000-1190.2015.04.004

[7] Zhang, S. (2013) Solutions to Diophantine Equation $\varphi(x y z)=4(\varphi(x)+\varphi(y)+\varphi(z))$. Journal of Northeast Petroleum University, 37, 113-118. https://doi.org/10.3969/j.issn.2095-4107.2013.06.016

[8] Yuan, H. and Li, P. (2018) The Positive Integer Solutions of Ternary Variable Coefficient Euler Function Equation $\varphi(a b c)=2 \varphi(a)+3 \varphi(b)+4 \varphi(c)$. Journal of Hubei University for Nationalities (Natural Science Edition), 36, 137-141. https://doi.org/10.13501/j.cnki.42-1569/n.2018.06.005

[9] Zhang, S. and Liu, Q. (2015) The Positive Integer Solutions of an Equation on the Euler Function. Journal of Northeast Normal University (Natural Science Edition), 47, 49-54. https://doi.org/10.16163/j.cnki.22-1123/n.2015.03.011

[10] Harrington, J. and Jones, L. (2010) On the Iteration of a Function Related to Euler's $\varphi$ Function. Integers, 10, 497-515. https://doi.org/10.1515/integ.2010.042 